\title{
EXPERIÊNCIA NO PROCESSO DE FORMAÇÃO NA CONSTRUÇÃO DE CONHECIMENTO SOBRE PREVENÇÃO DE QUEIMADURAS
}

\section{EXPERIENCE IN THE TRAINING PROCESS IN THE CONSTRUCTION OF KNOWLEDGE ABOUT BURN PREVENTION}

\author{
Rayhany Kelly de Sousa ${ }^{1 *}$ Matheus Scortegagna Leal ${ }^{2} *$ Marceli Cleunice Hanauer $^{3} *$ Natália $^{*}$ \\ Gonçalves $^{4}$
}

\section{RESUMO}

Objetivo: relatar a experiência de graduando e pós-graduandos em ministrar minicurso sobre prevenção de queimaduras em um evento de extensão de uma universidade federal. Método: relato de experiência. O material do minicurso foi elaborado por três estudantes e uma docente do curso de enfermagem, sendo duas enfermeiras, em formação de mestrado e um aluno do curso de graduação em enfermagem. Foram realizadas buscas na literatura científica e nas sociedades de que tratam assuntos de queimaduras e lesões de pele para o preparo do minicurso. Resultados: A atividade foi dividida em três momento e o material educativo foi composto por cinco blocos de conhecimentos, a saber: 1) Breve história sobre a queimaduras a partir do surgimento do fogo e o conceito de queimadura; 2) Epidemiologia no mundo e no Brasil; 3) Anatomia e Fisiologia da Pele; 4) Classificação e gravidade das queimaduras e por fim, 5) Os agentes causais e primeiros socorros. Conclusão: A construção, organização e apresentação do minicurso permitiu aos autores um aprendizado no que se diz respeito a identificação da necessidade do público, a procura por materiais relevantes sobre queimaduras e a transformação do conteúdo científico em uma linguagem acessível a todos.

Palavras-chave: Queimaduras; Educação em Enfermagem; Prevenção de Acidentes.

\begin{abstract}
Objective: to report on the experience of undergraduate and graduate students in a short course on the prevention of burns in a federal university extension event. Method: experience report. The material of the short course was prepared by three students, two nurses, in master's degree and one student in the undergraduate nursing course. Searches were realized out in the scientific literature and in the societies dealing with burns and skin lesions for the preparation of the mini-course. Results:The activity was divided into three stages and the educational material was composed of five blocks of knowledge, namely: 1) Brief history about burns from the onset of fire and the concept of burns; 2) Epidemiology in the world and in Brazil; 3) Anatomy and Physiology of the Skin; 4) Classification and severity of burns and, finally, 5) Causal agents and first aid. Conclusion: The construction, organization and presentation of the short course was important to the authors a learning a identification what the public needs, the search for relevant materials on burns and the text of the scientific content in a language accessible to everyone.
\end{abstract}

Keywords: Burns; Education, Nursing; Accident Prevention.

\footnotetext{
${ }^{1}$ Enfermeira. Mestranda do Programa de Pós-graduação em Enfermagem da Universidade Federal de Santa Catarina. Bolsista da Coordenação de Aperfeiçoamento de Pessoal de Nível Superior (CAPES). Membro do Laboratório de Pesquisas e Tecnologias em Enfermagem e Saúde a Pessoas em Condição Crônica. e-mail: rayhanyks@ gmail.com. ORCID: 0000-0002-0288-8192.

${ }^{2}$ Acadêmico do Curso de Graduação em Enfermagem da Universidade Federal de Santa Catarina. Bolsista do Programa Institucional de Bolsas de Iniciação Científica (PIBIC/CNPq). Membro do Laboratório de Pesquisas e Tecnologias em Enfermagem e Saúde a Pessoas em Condição Crônica. e-mail: enfermeirodermatologico@outlook.com. ORCID: 0000-0001-5653-6857.

${ }^{3}$ Enfermeira. Mestranda do Programa de Pós-graduação em Enfermagem da Universidade Federal de Santa Catarina. Membro do Laboratório de Pesquisas e Tecnologias em Enfermagem e Saúde a Pessoas em Condição Crônica. e-mail: tilitobinha@ gmail.com. ORCID: 0000-0002-5798-2709.

${ }^{4}$ Enfermeira. Doutora. Professora Adjunta do Departamento de Enfermagem e do Programa de Pós-graduação em Enfermagem da Universidade Federal de Santa Catarina. Membro do Laboratório de Pesquisas e Tecnologias em Enfermagem e Saúde a Pessoas em Condição Crônica. e-mail: nataliasjbv@gmail.com. ORCID: 0000-0002-9005-4381.
} 


\section{INTRODUÇÃO}

Os acidentes com queimaduras causam importante trauma e podem deixar sequelas, implicando em diferentes limitações físicas e psíquicas ${ }^{(1)}$. Apesar do número de acidentes por queimaduras causados por exposição a corrente elétrica, radiação, temperatura, exposição à fumaça, ao fogo e às chamas crescer nos últimos $10 \operatorname{anos}^{(2)}$ no Brasil, houve uma queda acentuada no número de acidentes no ano de $2020^{(3)}$, quando comparado com o ano de $2019^{(4)}$, o que pode estar relacionado ao isolamento social, o medo da ida ao pronto socorro e adquirir a infecção do Coronavírus e a subnotificações de $\operatorname{casos}^{(4-6)}$.

Grande parte desse tipo de acidente ocorre em domicílio ${ }^{(7)}$, local onde deve ser iniciado os primeiros socorros, a fim de minimizar o agravamento da lesão e sua sequela, para em seguida ser atendido em setores de emergência. Portanto, faz-se necessário a promoção da educação em saúde a fim de instruir sobre a prevenção e os primeiros socorros às pessoas que sofrem queimaduras e diminuir a gravidade das lesões ${ }^{(7-6)}$.

Considerando que na maioria dos casos, as queimaduras são acidentais e passíveis de prevenção, é de suma importância o compartilhamento de informações sobre seus fatores de risco e medidas de prevenção como uma estratégia para minimizar o número de sequelas, óbitos e acidentes relacionados a esse agravo ${ }^{(8-9)}$.

Na literatura, a prevenção pode ser definida por dois componentes: o passivo, no qual se incluem alterações no desenho e segurança de materiais e equipamentos; e o ativo, o qual elenca educação e informação. Os dois são importantes, mas destaca-se a educação, por se tratar de uma ferramenta transformadora na população em geral ${ }^{(10)}$.

Em estudo de Oliveira e Souza ${ }^{(8)}$ evidenciou-se a necessidade de campanhas educativas para população em geral, a fim de conscientizar as pessoas sobre a prevenção de queimaduras e acidentes domésticos, e ainda educação continuada nos mais diversos locais de trabalho.

Apesar da existência de políticas públicas e estratégias na Atenção Básica direcionadas à prevenção de acidentes, observa-se que essas ações ainda são incipientes, sendo poucas voltadas para a educação em saúde, devido ao déficit da equipe de saúde, em especial a enfermagem ${ }^{(12-13-14)}$. A falta de orientações adequadas aos pais, familiares e cuidadores, acarreta uma população desinformada e despreparada ${ }^{(13)}$.

O profissional da enfermagem bem como acadêmicos em enfermagem, tem a responsabilidade de participar da conscientização da população sobre os cuidados na prevenção de acidentes por queimaduras, para tanto ambos podem utilizar estratégias variadas, dentre estas podese destacar o desenvolvimento de cursos, cartazes informativos, oficinas entre outros ${ }^{(11)}$. 
Há necessidade dos profissionais de saúde serem mais proativos e promotores de informações, orientando os pais ou responsáveis sobre a prevenção de acidentes, discutindo este problema no mesmo nível de prioridade que os demais assuntos que envolvam o desenvolvimento saudável da criança, pois entende-se que a educação para comportamento em saúde deva iniciar já na infância. Destaca-se a importância de se abordar sobre prevenção de acidentes nas consultas médicas e de enfermagem durante a realização da puericultura; em ações preventivas e educativas nas escolas, e, até mesmo, desenvolver campanhas de conscientização e orientação sobre o assunto, a fim de disseminar conhecimento e reduzir agravos ${ }^{(16-17)}$.

Considerando essas estratégias de prevenção, no contexto da universidade, o tripé ensinopesquisa-extensão deve contemplar ações que possibilitem o acesso da sociedade às informações. A extensão universitária pode ser considerada como o espaço no qual teoria e prática se convergem como campo de formação em saúde, com aplicabilidade real para a população ${ }^{(15)}$, aliando esforços juntos à rede de saúde para promover comportamentos saudáveis, como na prevenção de acidentes por queimaduras.

Diante do exposto, este estudo teve como objetivo relatar a experiência de graduandos e pós-graduandos em ministrar um minicurso sobre a prevenção de queimaduras em um evento de extensão de uma universidade federal do Sul do Brasil.

\section{Relato da experiência}

Trata-se de um relato de experiência de graduando e pós-graduandos acerca da construção, organização e apresentação de um minicurso intitulado "Primeiros socorros e prevenção das queimaduras" no evento de extensão Semana de Ensino, Pesquisa e Extensão (SEPEX em casa), da Universidade Federal de Santa Catarina. Este minicurso estava vinculado ao projeto de extensão intitulado "Ações de Enfermagem para pessoas com feridas". Por se tratar de um evento aberto à comunidade, o público alvo foi todas as pessoas interessadas em conhecer a temática. Esta experiência aconteceu em dois dias no mês de outubro de 2020 através da plataforma Google meet.

A divulgação do minicurso se deu por meio do site oficial do evento e também por aplicativos de mensagens instantâneas, redes sociais para que as pessoas externas à universidade pública pudessem ter conhecimento do mesmo.

A atividade de apresentação do mini curso teve duração de duas horas, dividida em três momentos: 1) atividade "quebra gelo" (10 minutos) - na qual foi solicitado aos participantes que entrassem no site Menti e respondessem o que eles consideravam como queimaduras. Após, os 
resultados, compilados pelo próprio site, foram rapidamente analisados e, então, identificado o conhecimento dos participantes a esse respeito. Essa informação foi retomada ao longo da apresentação, quando os autores deste trabalho buscavam relacionar o conteúdo exposto com aquele analisado; 2) aula expositiva dialogada da temática; 3) momento para dúvidas, sugestões e comentários da construção e apresentação do material.

\section{Construção e organização do conteúdo}

A motivação em ministrar o curso na temática de queimaduras esteve associada a participação do acadêmico e pós-graduandos no Laboratório de Pesquisas e Tecnologias em Enfermagem e Saúde à Pessoas em Condição Crônica e projetos de extensão e pesquisa nos quais aborda-se a temática sobre queimaduras e a integra em suas discussões, eventos, e atividades práticas. Ainda, devido ao isolamento social, pensou-se que seria interessante disseminar os conhecimentos produzidos no contexto descrito acima para a população, considerando que as pessoas estavam passando maior tempo em casa, com risco aumentado de $\operatorname{acidentes}^{(18-19-20)}$.

Dessa forma, foi escolhida a temática "Primeiros Socorros e Prevenção de Queimaduras" para que assim, pudesse direcionar aos aspectos causadores no que se diz respeito à prevenção e como agir diante desses incidentes.

A partir disso, para a construção do material para o minicurso, os autores realizaram busca na literatura científica, manuais, diretrizes e também, em materiais que estavam voltados para leigos, para que assim pudessem ter um direcionamento de como abordar a temática de uma maneira clara e compreensível a todos os participantes.

No primeiro momento identificou-se que muito se aborda no Brasil sobre os tipos de queimaduras e os agentes causais. No entanto, os autores refletiram sobre a construção do conhecimento dos participantes em compreender os danos bio-fisico-psico-espirituais que permeiam a queimadura na vida de alguém e a repercussão desta.

Através dessa breve busca na literatura, os autores elegeram os possíveis tópicos para apresentação, divididos em blocos, a saber: 1) Breve história sobre a queimaduras a partir do surgimento do fogo e o conceito de queimadura; 2) Epidemiologia no mundo e no Brasil; 3) Anatomia e Fisiologia da Pele; 4) Classificação e gravidade das queimaduras e por fim, 5) Os agentes causais e primeiros socorros.

Como percebe-se, o bloco 1 foi construído para que o participante pudesse conhecer a importância de se falar sobre a temática desde os seus primórdios, pelo aumento significativo do 
trauma ao longo do tempo, registros históricos no Brasil sobre o tratamento e a definição da queimadura em si. Na figura 1, exemplo da tela deste bloco.

Figura 1 - Imagem dos registros históricos das queimaduras no Brasil.

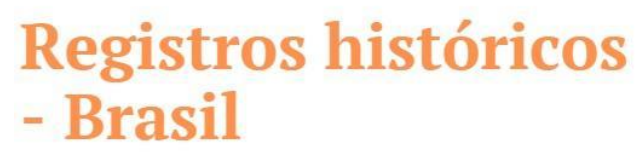

Em um estudo documental frente aos cuidados de enfermagem relacionados às feridas no Brasil no período de 1916 a 1946 , identificou-se os seguintes registros sobre as queimaduras:

\section{Primeiro grau}

Era utilizado pó de amido canforado, linimento, calcário, solução de bicarbonato de sódio, talco boricado ou compressas de água boricada fria.

\section{Terceiro grau}

\section{Segundo grau}

As bolhas não deviam ser rompidas, e sim drenadas por meio de uma punção asséptica flambada de agulha. Para isso, as bolhas e suas bordas eram preparadas com água e sabão, enxugadas e pinceladas com iodo a $2 \%$ ou mercúrio cromo.
Eram tratadas com compressas quentes embebidas em água, água boricada, solução de ácido tânico a 1 ou $4 \%$, diadermina boricada, solução saturada de ácido pícrico e sulfato de cobre, linimento de óleo calcário timolado

\section{Quarto grau}

Envolviam procedimentos radicais como a amputação de membros. Eram cuidadosamente limpas em ambientes antissépticos, por meio de lavagem com água e sabão esterilizados, aplicadas por meio de esponjas de gaze fina

Fonte: os autores ${ }^{(21)}$

No bloco 2, foram discutidos aspectos relacionados à epidemiologia. Para tanto, foi consultado o Sistema de Informações Hospitalares do SUS (DATASUS), nos anos de 2019 e 2020, considerando os grupos queimaduras e corrosões; outras causas externas de lesões acidentais; exposição a corrente elétrica, temperatura, pressão, radiação, exposição à fumaça, ao fogo às chamas, contato com fonte de calor e substância quentes. A análise foi pela morbidade hospitalar no SUS por local de internação (regiões do Brasil) $)^{(2-4)}$.

No terceiro bloco, no qual foi abordado sobre anatomia e fisiologia da pele, foi pensado para contribuir com o conhecimento sobre a importância da pele como órgão do corpo (Figura 2). Embora tenha sido mostrado termos técnicos, a linguagem utilizada na explanação foi pensada para o público em geral, sempre destacando que qualquer trauma na pele, poderia causar um desequilíbrio desse órgão e por isso, a importância de se cuidar e da prevenção de acidentes. 
Figura 2 - Imagem da tela sobre a importância da pele e suas funções.

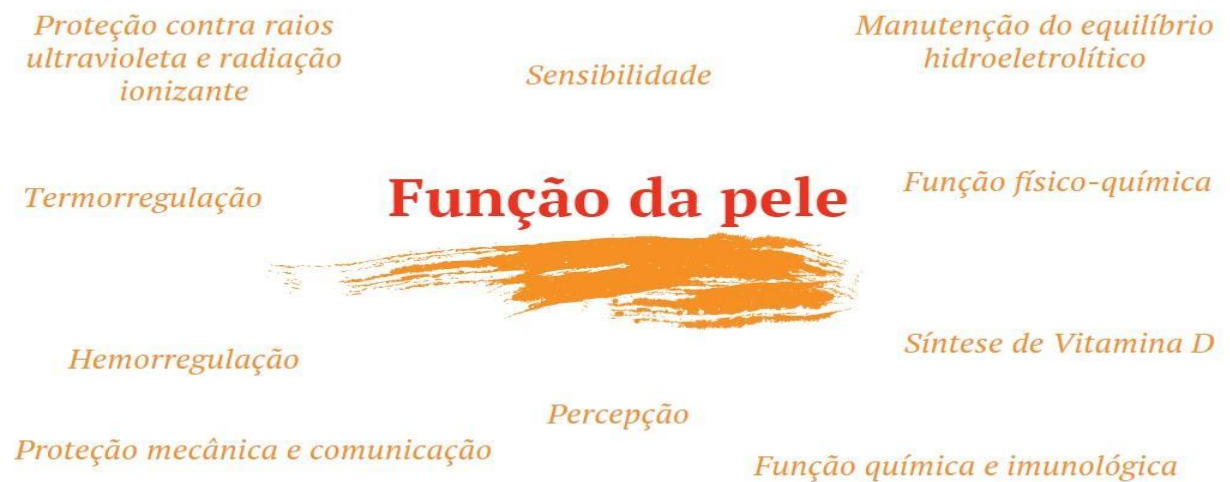

Fonte: os autores. ${ }^{(22)}$

Para a elaboração dos blocos quatro e cinco foram considerados os referenciais da Sociedade Brasileira de Queimaduras e da American Burn Association. Para ampliar o conhecimento acerca de quando procurar um serviço de saúde quando sofrer uma queimadura, foi explanado sobre a classificação das lesões (primeiro, segundo e terceiro grau), como os profissionais avaliam a extensão e quais os critérios utilizados para o encaminhamento a serviços especializados.

Após, no último bloco, foram tratados de algumas causas de queimaduras, a saber: 1) Queimaduras por produtos inflamáveis; 2) Queimaduras por líquidos quentes; Queimadura solar; 3) Queimaduras Químicas; 4) Queimadura por plantas; 5) Queimaduras por cnidários; 6) Queimaduras solar; 7) Queimadura elétrica. Ainda, foram abordados sobre a prevalência, os cenários de riscos com cada um deles, fatores agravantes, os cuidados e as intervenções que podem ser realizadas para prevenir/socorrer o indivíduo.

Todos os autores ficaram responsáveis em buscar na literatura e a construção do material ocorreu em conjunto, utilizou-se do Google Drive para compartilhamento das informações e a escrita do material de forma simultânea através da ferramenta Google Docs.

Durante a construção do material, foram mediadas várias reuniões entre os autores para que pudessem delinear o conhecimento, pois tinha-se como desafio: trazer o conhecimento de forma acessível para todos que se faziam presentes, abordar sobre os tópicos elencados e ainda, em um tempo de duas horas. 
Dessa forma, os autores utilizaram como estratégia a identificação do conhecimento através dos tópicos, elencaram os principais pontos, ou seja, abordando os termos científicos mas explicando de forma simplificada o seu significado/funcionalidade. Como também, integrando o conhecimento de forma contextualizada com a vida diária do participante, como por exemplo, ao fritar uma comida, a cafeteira italiana, as notícias de queimaduras com álcool em gel decorrente da proximidade com o fogo, entre outros exemplos em que fizesse entender o processo que é desencadeado o acidente por queimadura.

E dessa forma, foi construída a apresentação, em que foi utilizado a ferramenta online e gratuita Canva no qual permitiu a utilização de imagens e animações para elaboração de um conteúdo interativo e simplificado, no total foram 72 slides.

\section{DISCUSSÃO}

A atividade foi dividida em três momentos, sendo o primeiro destinado a identificar o conhecimento dos participantes acerca da temática, este processo foi mediado por um aplicativo online e gratuito no qual oportuniza que o participante responda uma pergunta com palavras chaves, sendo a pergunta central “O que são queimaduras?”, para que assim os autores pudessem identificar os conceitos ou até mesmo explorá-los durante a condução do minicurso. As respostas citadas com maior número de vezes apresentaram-se com letras maiores e aquelas que foram citadas com menor número apresentaram-se em tamanhos menores.

O segundo momento da atividade foi a apresentação propriamente dita, em que foi construído apresentando os aspectos históricos, aspectos epidemiológicos no Brasil e no mundo, as respectivas estruturas da pele e suas funcionalidades, conceito de queimaduras, a relação de temperatura do agente causal e a classificação da lesão quanto ao tipo de lesão, como também sobre a disposição dos serviços de saúde presentes na Rede de Atenção à Saúde que contemple o atendimento ao paciente com lesão queimada, sendo, a Atenção Primária à Saúde, Atenção de Urgência e Emergência como Unidades de Pronto Atendimento e Hospitais, como também o Centro de Tratamento de Queimados (CTQ) e seus respectivos critérios.

Para o melhor compreendimento da Atenção à Saúde envolvida ao paciente queimado, foi explanado sobre os tipos de queimaduras, sendo, Queimadura de Primeiro Grau, Queimadura de Segundo Grau Superficial, Queimadura de Segundo Grau Profunda e Queimadura de Terceiro Grau, informando as características de cada uma e os cuidados necessários. Expondo aos participantes de https://doi.org/10.31011/reaid-2021-v.95-n.35-art.1140 Rev Enferm Atual In Derme v. 95, n. 35, 2021 e-021113 
forma contextualizada com a realidade, no que se diz respeito às atividades domésticas, colaborativas e sociais, e assim, dispondo o tipo de cada atendimento de cada serviço de saúde disposto a atender o paciente queimado dependendo da sua gravidade.

E assim, subsidiou-se o espaço para também abordar sobre a extensão para determinação da Superfície Corporal Queimada, em que é um dos aspectos que determinam a classificação da gravidade da queimadura em conjunto ao Tempo de Exposição, Comorbidades do Paciente, Idade e o Agente ${ }^{(23)}$.

Frente a isto, existem vários agentes causais para a ocorrência da queimadura, de acordo com a literatura internacional são: escaldo, chama, raios, fogos de artifício, elétrica, contato, química, relacionada ao trabalho e hospitalares ${ }^{(24)}$.

Para compreender tais fatores e adequar a realidade brasileira, foram realizadas buscas em estudos brasileiros que abordassem sobre os agentes causais de acordo com a prevalência nacional, no qual foi encontrado em que foi visto: as queimaduras por agentes inflamáveis (álcool, gasolina, óleo de cozinha, querosene e solventes ${ }^{(25)}$, as queimaduras por chamas ${ }^{(26-27)}$, as queimaduras por líquidos quentes ${ }^{(28)}$, as queimaduras químicas por soda cáustica ${ }^{(29-30)}$, por ácido fluorídrico ${ }^{(31)}$, por fenol $^{(32)}$, por plantas como limão, laranja e toranja ${ }^{(33)}$ e por cnidários ${ }^{(34)}$. Como também, as queimaduras por radiação solar ${ }^{(35)}$, queimaduras por eletricidade ${ }^{(36)}$, e por fim, a queimaduras pela eletrocirurgia $^{(37)}$.

Nessa perspectiva, abordou-se os fatores de riscos condizentes com os aspectos ambientais, sociais e econômicos presentes na realidade do Brasil, visto que estes estão diretamente relacionados com a epidemiologia. Como por exemplo, o aumento do gás, muitas pessoas começaram a procurar por outras fontes de geração de fogo como os líquidos inflamáveis para cozinhar ${ }^{(38)}$, como também uma grande região litorânea do país, apresenta maior prevalência de queimaduras solares e por animais marítimos como os cnidários.

Como também decorrente ao período de pandemia propriamente dito, em que apresentou-se maior número de acidentes com crianças em domicílio(18) decorrente do isolamento social, queimaduras por álcool decorrente da necessidade de higienização das mãos para prevenção da COVID-19(39).

No geral, para cada queimadura foi explanado a epidemiologia, os fatores de riscos, gravidade das lesões e a prevenção, sendo esta última relacionada ao indivíduo e ao coletivo por meio de ações que poderiam ser mediadas nas instituições, como por exemplo não se aproximar do 
fogo após higienizar as mãos com álcool em gel e divulgar nas praias onde há predominância dos cnidários para evitar os acidentes.

No final da explanação do conteúdo também foi abordado sobre as situações mais comuns no cotidiano em que tornam-se uma situação de perigo, sendo, acender a churrasqueira com álcool, produzir sabão líquido caseiro com mistura de hidróxido de sódio ou soda cáustica $\mathrm{NaOH}$, manipular fiação elétrica de baixa e alta tensão sem o devido preparo técnico, atear fogo em entulhos domésticos com gasolina ou outro líquido inflamável, manipular frituras com grandes quantidades de óleo, o uso de cafeteira do tipo italiana, descer pelo lado do escapamento da moto e manipular óleo fervente e deixar água cair ${ }^{(40)}$.

E se acontecer esses acidentes, foi ressaltado os cuidados que devem ser tomados como o resfriamento da área lesada e cobrir com um pano limpo (de preferência úmido), cobrir o indivíduo com cobertas quando a queimadura for extensa para evitar a hipotermia, como também, retirar os adornos, lentes de contato e roupa (se estas não se apresentarem aderidas) e por fim, ressaltado procurar por um profissional ou serviço de saúde para avaliação da lesão e o devido tratamento ${ }^{(40)}$.

Foi um momento oportuno para desmistificar alguns aspectos e conceitos culturais no que se diz respeito ao tratamento caseiro das queimaduras, como utilizar pasta de dente, pimenta, gelo, ovo, entre outros produtos de qualquer origem que não seja de uso comprovado para o tratamento dessas lesões, como também ações imediatas como evitar correr para que a produção do fogo não se acentue e evitar estourar as bolhas ${ }^{(40)}$.

O terceiro e último momento da atividade foi a abertura para os participantes para explanar suas dúvidas, comentários e sugestões sobre a temática e a atividade propriamente dita. O que tornou-se um momento oportuno e de construção coletiva, pois estavam-se presentes profissionais de saúde e estudantes em que aproveitaram para perguntar sobre a melhor conduta em relação às bolhas, sobre as formas de tratamento das queimaduras e se apresentaram surpreendidos em relação às plantas que causam queimaduras. Também foi abordado pelos participantes sobre a predominância de cnidários em determinadas praias.

No geral, a apresentação foi elogiada pelos participantes em relação a pertinência, clareza e domínio do conteúdo pelos participantes, nos dois dias a atividade contemplou a presença de 28 participantes. Como também, os autores foram honrados com a presença do Presidente da Sociedade Brasileira de Queimaduras, o qual propôs a construção de um projeto para semear a temática pelos autores, tornando assim, um momento de expectativa e entusiasmo. 


\section{CONCLUSÃO}

A construção, organização e apresentação do minicurso permitiu aos autores um aprendizado no que se diz respeito: identificação do problema, ou seja, qual a necessidade do público, a procura por materiais relevantes sobre queimaduras e a transformação do conteúdo científico em uma linguagem acessível a todos.

\section{REFERÊNCIAS}

1. Gonçalves N. et al. Cuidado de enfermagem ambulatorial em pessoa com queimadura elétrica: relato de caso. 2020;94(32):e-20083.

2. Departamento de Informática do SUS - TABNET/DATASUS. Ministério da Saúde. Brasil. Acidentes por queimaduras, exposição a corrente elétrica, radiação, temperatura, exposição à fumaça, ao fogo e às chamas: de 2010 à 2020. Disponível em: http://tabnet.datasus.gov.br/cgi/tabcgi.exe?sih/cnv/fiuf.def. Acesso em: 20 fev. 2021.

3. Departamento de Informática do SUS - TABNET/DATASUS. Ministério da Saúde. Brasil. Acidentes por queimaduras, exposição a corrente elétrica, radiação, temperatura, exposição à fumaça, ao fogo e às chamas: 2020. Disponível em: http://tabnet.datasus.gov.br/cgi/tabcgi.exe?sih/cnv/fiuf.def. Acesso em: 20 fev. 2021.

4. Departamento de Informática do SUS - TABNET/DATASUS. Ministério da Saúde. Brasil. Acidentes por queimaduras, exposição a corrente elétrica, radiação, temperatura, exposição à fumaça, ao fogo e às chamas: 2019. Disponível em: http://tabnet.datasus.gov.br/cgi/tabcgi.exe?sih/cnv/fiuf.def. Acesso em: 20 fev. 2021.

5. Marcheti MA, et al. Acidentes na infância em tempo de pandemia pela COVID-19. Rev Soc Bras Enferm Ped. 2020;20(Especial COVID-19):16-25.

6. Blank D, Waksman RD. A importância das injúrias por acidentes domésticos em tempos de COVID-19. Resid Pediatr. 2020;10(2):1-6.

7. Castro RR, Ferreira AS, Santos SSA. Atendimento ao queimado em uma unidade de saúde pública. Rev Educação em Saúde. 2020;8(1):3-11.

8. Souza MCA, Oliveira LCF. Fatores de risco de queimaduras domésticas: conhecimento de profissionais de Unidades Estratégia de Saúde da Família. Revista de Saúde. 2019;10(1):9-14.

9. Swiderski JS, Takemoto AY. Assistência de enfermagem em crianças vítimas de queimaduras: uma revisão integrativa da literatura. [Trabalho de Conclusão de Curso]. Guarapuava (PR). Repositório Institucional Uni Guairacá. 2019

10. Pitol DN. Haddad A. Educational Children's Storybook for Burn prevention during childhood. [Dissertação de mestrado]. São Paulo (SP). Universidade Federal de São Paulo (UNIFESP). 2018.

11. Rocha LN. et al. A educação em saúde sobre primeiros socorros e prevenção de acidentes na escola: uma revisão integrativa de literatura. Rev Liberum Accessum. 2020;2(1):1-16.

12. Brito MA. et al. Fatores de risco no ambiente no ambiente doméstico para quedas em crianças menores de cinco anos. Rev Gaúcha de Enfermagem. 2017;38(3):e2017-0001. 
13. Faria CGD, et al. Principais causas de internação por acidente domésticos na infância em um hospital universitário do oeste do Paraná. Brazilian Journal of Surgery and Clinical Research. 2018;22(2):103-9.

14. Silva JDS, Fernandes KDS, Pereira D. Acidentes domésticos mais frequentes em crianças. [Trabalho de Conclusão de Curso]. Brasília (DF). Centro Universitário do Planalto Central Apparecido dos Santos. 2019.

15. Costa ACDPD, et al. Educação e Saúde: a extensão universitária como espaço para tencionar e pensar a educação em saúde. Brazilian Journal Of Development. Curitiba. 2020;6(4):15 .

16. Araújo AR, et al. Prevenção de acidentes em uma creche: experiência com pais, professores e pré-escolares. Rev. de Enfermagem UFPE online. 2017;11(4):1671-78.

17. Filócomo, et al. Perfil dos acidentes na infância e adolescência atendidos em um hospital público. Acta Paulista de Enfermagem. 2017;30(3):287-94.

18. Sociedade Brasileira de Pediatria. Prevenção de queimaduras em tempos de COVID-19 [Internet]. Brasília. 2020 [Citado em 2021]. Disponível em: https://www.sbp.com.br/fileadmin/user_upload/22630b-NA_-

_Prevencao_Queimaduras_tempos_Covid19.pdf

19. Secretaria de Estado de Saúde. Casos de queimaduras com líquidos quentes aumentam $41 \%$ no HEA [Internet]. Alagoas. 2020 [Citado em 2021]. Disponível em: https://www.saude.al.gov.br/casos-de-queimaduras-com-liquidos-quentes-aumentam-41-no-he-doagreste/

20. Agência Minas Gerais. Especialistas alertam para tendência de aumento nas queimaduras por álcool [Internet]. Minas Gerais. 2020 [Citado em 2021]. Disponível em: http://www.agenciaminas.mg.gov.br/noticia/especialistas-alertam-para-tendencia-de-aumento-nasqueimaduras-por-alcool

21. Vieira RQ et al. Primeiros escritos sobre os cuidados de enfermagem em feridas e curativos no Brasil (1916-1947). Hist. Enfer., Rev Eletrônica. 2017;8(2):106-17.

22. Domansky RC. Borges, EL Manual para prevenção de lesões de pele: Recomendações Baseadas em Evidências. Rio de Janeiro: Rubio, 2012. 288p.

23. Malta DC, et al. Perfil dos casos de queimadura atendidos em serviços hospitalares de urgência e emergência nas capitais brasileiras em 2017. Revista Brasileira de Epidemiologia. 2020;23(1):1-14.

24. Herdon DN. Total Burn Care. 5. ed. Texas: Elsevier, 2018.

25. Nestor A, Turra K. Perfil epidemiológico dos pacientes internados vítimas de queimaduras por agentes inflamáveis. Rev Bras Queimaduras. 2014;13(1):44-50.

26. Oliveira RC, et al. Trauma por queimaduras: uma análise das internações hospitalares no Brasil. Rev Eletrônica Acervo Saúde. 2020;12(2):1-9.

27. Gonçalves AJ, Cunha MTRD, Santos-Júnior JFD. Estudo epidemiológico das queimaduras no Hospital de Clínicas da Universidade Federal do Triângulo Mineiro. Rev Bras. Cir. Plást. 2020;35(4):420-6.

28. Oliveira WCM, et al. Fatores de risco e medidas de prevenção das queimaduras infantis: revisão integrativa da literatura. Rev Brasileira de Queimaduras. 2016;15(4):267-73.

29. Teles GGDA, et al. O uso da abdominoplastia reversa para o tratamento de queimadura química em região toracoabdominal. Rev Bras. Cir. Plastica. 2020;35(1):113-7. 
30. Santos D, Nagashima L. Saber popular e o conhecimento científico: relato de experiência envolvendo a fabricação de sabão caseiro. Rev de Ensino de Ciências e Matemática. 2017;8(2):12742.

31. Guedes V, et al. Etiologia e tratamento de queimaduras provocadas por agente químico: scoping review. Rev ROL de Enfermería. 2020;43(1):129-38.

32. Bello HRS, Santos FSD, Correa CR. Cuidados de enfermagem ao paciente queimado. 1. ed. Porto Alegre: Moriá, 2018.

33. Bravo BSF, Balassiano LKA. Fitofotodermatite por Ruta graveolens com manifestação cutânea extensa. Rev Bras Queimaduras. 2014;13(3):177-9.

34. Santo-Aquino GGDE, Junior VH, De-Albuquerque-Pires V. Avaliação dos acidentes ocorridos por cnidários no município de Salinópolis/Pará (Brasil). Biota Amazônia (Biote Amazonie, Biota Amazonia, Amazonian Biota), 2020;9(4):37-40.

35. Mendes DCL, Cruz 1. Como otimizar o cuidado interprofissional do paciente de alta complexidade com recuperação de queimaduras por meio da prática de enfermagem baseada em evidência? Journal of Specialized Nursing Care. 2020;12(1):3277.

36. Secretaria de Estado da Saúde. Médica da Unidade de Tratamento de Queimados do Huse alerta para queimaduras elétricas [Internet]. Sergipe. 2019 [Citado em 2021]. Disponível em: https://www.saude.se.gov.br/?p=30939

37. Bisinotto FMB, et al . Queimaduras relacionadas à eletrocirurgia - Relato de dois casos. Rev Brasileira de Anestesiologia. 2017;67(5):527-34.

38. Rodrigues MF, et al. Relação do preço do gás de cozinha e queimaduras por líquido inflamável. Rev Bras Queimaduras. 2019;18(3):162-66.

39. Sociedade Brasileira de Pediatria. Uso do álcool em gel aumenta acidentes com queimaduras [Internet]. Brasil. 2020 [Citado em 2021]. Disponível em: https://www.sbp.com.br/imprensa/detalhe/nid/uso-do-alcool-em-gel-aumenta-acidentes-comqueimaduras/\#: :text=\%E2\%80\%9CN\%C3\%A3o\%20existe\%20raz\%C3\%A3o\%20para\%20usar,al erta\%E2\%80\%9D\%2C\%20afirmou\%20a\%20dra

40. Sociedade Brasileira de Queimaduras. Prevenir para evitar: manual de prevenção de queimaduras. Manual de Prevenção de Queimaduras [Internet]. 2018 [Citado em 2021]. Disponível em: https://sbqueimaduras.org.br/material/1331

Submissão: 2021-07-28

Aprovado: 2021-06-12 